\title{
Impacto de la contaminación orgánica sobre el fitoplancton de un arroyo de la llanura pampeana
}

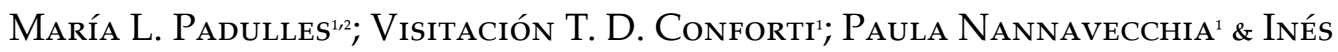 \\ O'FARRELL ${ }^{2, \mathbb{}}$ \\ ${ }^{1}$ Departamento de Biodiversidad y Biología Experimental, Facultad de Ciencias Exactas y Naturales, Universidad de Buenos \\ Aires - Instituto de Biodiversidad y Biología Experimental y Aplicada (IBBEA) (CONICET-UBA). Buenos Aires, Argentina. \\ ${ }^{2}$ Departamento de Ecología, Genética y Evolución, IEGEBA (CONICET-UBA), Facultad de Ciencias Exactas y Naturales,
} Universidad de Buenos Aires.

\begin{abstract}
RESUMEN. Este trabajo analiza la influencia de la calidad del agua sobre el fitoplancton y, en particular, sobre el ensamble de euglenofitas de un arroyo periurbano de la provincia de Buenos Aires (Argentina), muy afectado por la recepción de aguas residuales urbanas e industriales. Los índices de calidad de agua ICA (Berón 1984) calculados sobre el cauce principal y sus afluentes mostraron un deterioro marcado de las aguas y evidenciaron un claro patrón espacial. Además, en más de un sitio muestreado, muchos de los valores de los parámetros indicadores de la calidad del agua para la preservación de la vida acuática excedieron los rangos establecidos por las normas nacionales. El fitoplancton estuvo dominado por clorofitas, con codominancia de cianobacterias y euglenofitas. Se identificaron las especies de euglenofitas y se evaluó la presencia de deformaciones morfológicas, que fueron asociadas a la contaminación orgánica. Dada la importancia de las comunidades autotróficas en arroyos de llanura, se sugiere implementar el uso de los ensambles de euglenofitas como centinelas de los cambios producidos por el exceso de materia orgánica característico de los cursos urbanizados.
\end{abstract}

[Palabras clave: Euglenophyta, arroyo periurbano, calidad de agua, aguas residuales industriales]

\begin{abstract}
Aвstract. Organic pollution impact on the phytoplankton of a stream of the Pampas plains. This paper analyzes the influence of water quality on phytoplankton, focusing on the euglenoid assemblages of a periurban stream in the province of Buenos Aires (Argentina), strongly impacted by urban and industrial wastewater. The ICA water quality index (Berón 1984) calculated for the main channel and its tributaries showed a marked deterioration of the waters and evidenced a clear spatial pattern. In addition, in some of the sampled sites, water quality indicators exceeded the ranges established by national standards. The phytoplankton was dominated by chlorophytes, with codominance of cyanobacteria and euglenophytes. Euglenophyta species were identified and the occurrence of morphological anomalies was associated and evaluated in relation with organic pollution. Given the importance of autotrophic communities in lowland streams, we suggest the use of euglenophyte assemblages as sentinels of changes produced by the excess of organic matter, which is characteristic of urban watercourses.
\end{abstract}

[Keywords: Euglenophyta, peri-urban stream, water quality, industrial wastewater]

\section{INTRODUCCIÓN}

El crecimiento poblacional de los grandes centros urbanos a causa de la inmigración de la población rural genera zonas densamente habitadas, las que, sumado al incremento y a la reubicación de la actividad industrial, constituyen factores de gran impacto sobre los suelos y cuerpos de agua aledaños. En particular, los cursos de agua se ven alterados por el gran caudal de agua que las industrias y los centros urbanos extraen y, eventualmente, vierten sin tratamiento previo (UNESCO 2006). Los ecólogos describieron un síndrome de arroyos urbanizados que presenta varios atributos, entre los cuales se incluye el exceso de nutrientes y contaminantes, el aumento de los pulsos hidrológicos momentáneos y las alteraciones en los ensambles bióticos (Meyer et al. 2005).

Editora asociada: María Diéguez
En la Argentina, estos fenómenos se pueden observar principalmente alrededor de las grandes urbes. Los arroyos de la región pampeana se caracterizan por la ausencia de vegetación riparia, por bajas velocidades de corriente y por ser receptores de una cantidad elevada de efluentes industriales y domésticos (Feijoó and Lombardo 2007). En la zona Metropolitana del Gran Buenos Aires, la población y las industrias se concentran alrededor de tres cuencas de caudal considerable y de gran superficie: Matanza-Riachuelo, Reconquista y Luján. Alrededor de esta última se ubica el 3.6\% de los establecimientos industriales del país (Herrero and Fernández 2008). Momo et al. (2003) indicaron que esta cuenca presentaba una contaminación moderada, con un aumento del deterioro en dirección aguas abajo, en coincidencia con la confluencia de

Recibido: 26 de abril de 2017

Aceptado: 23 de agosto de 2017 
los afluentes más deteriorados (Feijoó et al. 1999). Muchos de estos afluentes atraviesan zonas industriales. En particular, en la zona media del Arroyo Carabassa, varias industrias alimentarias realizan descargas de gran envergadura, que contaminan el agua con altos tenores de materia orgánica. A su vez el arroyo atraviesa zonas agrícolas, campos de golf y recibe por escorrentía en forma de contaminación difusa agroquímicos tales como fertilizantes. Diferentes estudios realizados en la zona (Momo et al. 2003; Plataroti 2010) consideraron el estado ecológico de este arroyo como muy malo, y recientemente un estudio de los sedimentos en la desembocadura del Arroyo Carabassa concluyó que éstos son peligrosos para la biota acuática (Peluso et al. 2016).

Los cuerpos de agua contaminados del conurbano bonaerense presentan ensambles fitoplantónicos característicos formados por organismos que se ven favorecidos o toleran concentraciones altas de materia orgánica (Gómez and O’Farrell 2014). La mayoría de las especies de microalgas euglenofitas incrementan su desarrollo en aguas ricas en materia orgánica, preferiblemente con compuestos ferrosos y sales de amonio (Conforti 1998). Su importancia en ambientes alterados por grandes concentraciones de estos contaminantes permite deducir que muchas especies podrían tolerar estas condiciones. A su vez, el exceso de materia orgánica disuelta en estos ambientes genera una gran proliferación bacteriana. Estos organismos liberan metabolitos (e.g., vitaminas) que favorecen el desarrollo de euglenofitas en cuerpos de agua con estas características. Por otro lado, estudios realizados sobre Lepocinclis acus y Phacus brachykentron demostraron que existe una relación entre la concentración de materia orgánica y la deformación de la morfología celular original (Conforti 1998; Bauer et al. 2012; Nannavecchia et al. 2014; Nannavecchia 2016).

El objetivo de este estudio fue evaluar los efectos de la degradación del Arroyo Carabassa, asociada a las descargas de las industrias localizadas en la vera del cauce principal o de sus afluentes, sobre el fitoplancton y las características del ensamble de euglenofitas. Se hipotetiza que el deterioro de la calidad del agua debida a los excesos de desechos orgánicos afecta la estructura del fitoplancton y en particular, a los atributos morfo-fisiológicos de los ensambles de euglenofitas.

\section{Materiales y Métodos}

\author{
Área de estudio
}

El Arroyo Carabassa forma parte de la cuenca del Río Luján, que se extiende en sentido SO$\mathrm{NO}$ en la provincia de Buenos Aires y abarca una superficie de $2690 \mathrm{~km}^{2}$ (Figura 1). Se distinguen tres zonas: superior $(40 \mathrm{~km}$ de longitud), media $(30 \mathrm{~km})$ e inferior $(60 \mathrm{~km})$ (O'Farrell et al. 2002). Este curso se encuentra en la transición entre la zona media y baja, en un contexto de gran densidad poblacional e intensa actividad industrial (Giorgi 2001; Rodríguez et al. 2008). Su naciente se localiza luego de la confluencia de dos arroyos, se extiende en sentido NE por $5.2 \mathrm{~km}$ y desemboca en el Río Luján (Figura 1). El clima de la zona es templado y húmedo, con temporada seca en invierno y lluvias desplazadas hacia el otoño, con una media anual de $950 \mathrm{~mm}$. La temperatura media oscila entre 22 y $10{ }^{\circ} \mathrm{C}$ (Fidalgo 1983).

\section{Metodología del muestreo}

Se tomaron muestras en seis sitios: tres ubicados sobre diferentes afluentes (AfA, AfB y AfC) y tres a lo largo del curso principal (S1, S2, S3) del Arroyo Carabassa. AfA se estableció sobre el curso de agua que recibe las descargas de los piletones de la planta de tratamiento para aguas residuales de una industria láctea, AfB aguas abajo de un frigorífico y AfC luego de un barrio privado. En el curso principal se establecieron los sitios S1, ubicado 500 m río abajo de la confluencia de los afluentes AfA y AfB, que dan origen al arroyo, S2 en un barrio privado luego de la desembocadura de AfC, y S3 aguas abajo de S2 luego de la descarga de una planta de la industria alimentaria.

Los muestreos se realizaron el 20/10/2011, el 25/4/2012 y el 21/9/2012. En cada punto se colectaron muestras de fitoplancton subsuperficiales para el análisis cualitativo utilizando una red de $15 \mu \mathrm{m}$ de poro; una se fijó con formol al 3\% y otra fue mantenida viva. Para la cuantificación del fitoplancton se tomaron muestras de $250 \mathrm{~mL}$ y se fijaron con solución lugol. In situ se midió la velocidad, la profundidad y el ancho del curso de agua. Se determinaron la temperatura, el pH y la conductividad con un potenciómetro y un conductímetro Hanna Instruments, y el oxígeno disuelto (OD) con un oxímetro Oxi 330/SETWTW. La transparencia se registró mediante un disco de Secchi. Se determinó la turbidez por el método nefelométrico; la concentración de nitratos $\left(\mathrm{N}_{-} \mathrm{NO}_{3}^{-}\right)$, por reducción 
de cadmio; de nitritos $\left({\mathrm{N}-\mathrm{NO}^{-}}_{2}\right)$, por método colorimétrico; amonio $\left(\mathrm{N}-\mathrm{NH}_{4}^{+}\right)$, por electrodo de ion selectivo de amonio; nitrógeno total (NT), por Macro-Kjeldahl y volumetría, y el fósforo total (PT), por digestión persulfatoácido ascórbico. Los sólidos suspendidos totales (SST) se determinaron por el método gravimétrico $103-105{ }^{\circ} \mathrm{C}$; las sustancias fenólicas, por extracción con cloroformo y fotométrico directo; los tensoactivos, por sustancias activas al azul de metileno (SAAM); la materia orgánica, por la demanda biológica de oxígeno (DBO) (incubando 5 días); la demanda química de oxígeno (DQO), en reflujo cerrado y colorimetría, y las bacterias coliformes totales y fecales por el número más probable (NMP). Las DBO y DQO son análisis utilizados de rutina para evaluar la cantidad agregada de materia orgánica en agua y efluentes (APHA 2005). Las determinaciones se realizaron siguiendo las normas de la American Public Health Association (APHA 2005).

Las variables fueron comparadas con los niveles guía de calidad de agua ambiente para la preservación de la vida acuática nacionales (SRHN 2005) y, en caso de no existir normativa nacional, se utilizaron criterios internacionales (DOF 1989; USEPA 1999).

Los resultados obtenidos se utilizaron para determinar el índice de calidad de agua ICA (Berón 1984), y se consideraron las concentraciones $\left(\mathrm{q}_{\mathrm{i}}\right)$ de las siguientes variables, con sus respectivos pesos $\left(\mathrm{w}_{\mathrm{i}}\right)$ : $\mathrm{DBO}, \mathrm{w}=3$; amonio, $\mathrm{w}=3$; $\mathrm{OD}, \mathrm{w}_{\mathrm{OD}}=2$; temperatura $\mathrm{w}=1$. Este índice permite relacionar las variables ambientales y obtener una caracterización de la calidad del agua; varía entre $0 \leq \mathrm{ICA} \leq 10$. Los valores de ICA entre 0 y 3 corresponden a polución muy elevada; entre 3 y 6 , a polución elevada, entre 6 y 8, a polución intermedia, y 10 corresponde a la pureza original.

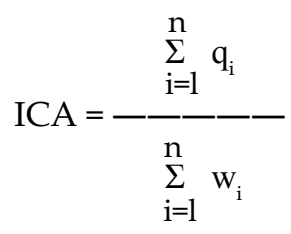

$\mathrm{q}_{\mathrm{i}^{\prime}}$ concentración y $\mathrm{w}_{\mathrm{i}}$ peso relativo de la variable $\mathrm{i}$

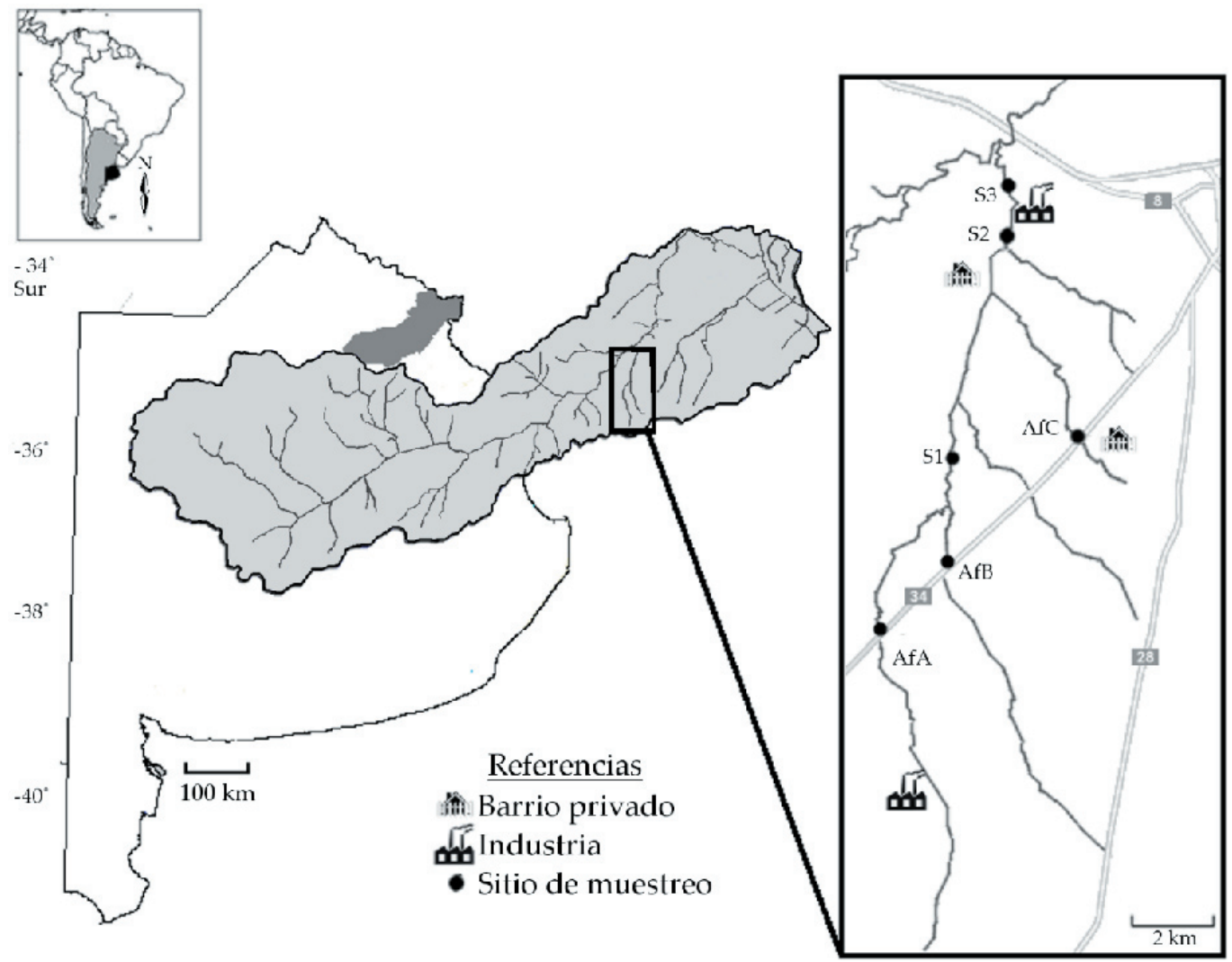

Figura 1. Ubicación del área de estudio en la cuenca del Río Luján. Se detallan los sitios de muestreo en el Arroyo Carabassa y sus tributarios.

Figure 1. Map showing the location of the study area in the Luján River with a detail of the sites at the Carabassa stream and its tributaries. 
En cada sitio se examinó la flora de las microalgas euglenofitas. El análisis de las muestras vivas evita que se produzcan alteraciones morfológicas por manipulación o efecto de los fijadores, lo que posibilita observar caracteres de importancia taxonómica durante la natación de las algas. Las euglenofitas de las muestras del 21/9/2012 fueron fotografiadas y cuantificadas para analizar la existencia de alteraciones frente a su morfología habitual. La determinación se realizó usando la bibliografía pertinente (Hüber-Pestalozzi 1955; Popova 1966; Starmach 1983; Tell and Conforti 1986; Conforti 1999; Conforti and Joo 1994; Kim et al. 1999; Wolowski and Hindák 2005; Ciugulea and Triemer 2010). El recuento de fitoplancton total se realizó bajo microscopio invertido (Utermöhl 1958), y se discriminaron las clases más representativas. El error estimado no superó el 20\% (Venrick 1978).

Se realizó un ordenamiento de los sitios correspondientes a las distintas fechas por medio de un análisis de componentes principales (ACP) con las variables hidrológicas, físicas y químicas no altamente correlacionadas $(\mathrm{r}<0.8, P<0.05)$ : caudal, SST, temperatura, DQO, OD, nitratos, nitritos, amonio, NT, PT y SAAM. Se realizaron correlaciones paramétricas de a pares con el índice de Pearson entre la abundancia de euglenofitas y las variables físicas y químicas. Los análisis estadísticos se realizaron con el programa INFOSTAT 2013. La abundancia de euglenofitas y su proporción sobre el fitoplancton total se correlacionaron con una nueva variable descriptiva de la calidad del agua del sistema, obtenida a partir del mayor autovalor del ACP (los "scores" de los sitios en el primer eje del ACP).

\section{Resultados}

\section{Variables físicas y químicas}

En la Tabla 1 se presentan los promedios de las variables hidrológicas, físicas y químicas de los sitios de muestreo. El caudal en los afluentes sólo pudo calcularse para el sitio AfA, que mostró un caudal medio de $0.21 \mathrm{~m}^{3} / \mathrm{s}$ (Tabla 1). Este curso produjo el mayor aporte de agua al cauce principal del arroyo. El escaso flujo de agua en AfB y AfC no permitió cuantificar sus respectivos caudales con la metodología usada. En el cauce principal el caudal fue máximo en S1 durante octubre del 2011 (0.9 $\mathrm{m}^{3} / \mathrm{s}$ ) y disminuyó aguas abajo. Los máximos se correspondieron con las precipitaciones más abundantes, ocurridas durante la primavera (setiembre 2012), y los menores caudales se detectaron en abril de 2011, en coincidencia con la época de menor precipitación, luego de un período cálido (Figura 2).

Los parámetros turbidez, conductividad, DBO, DQO, SST, SAAM y PT presentaron en la mayoría de los muestreos un patrón espacial, con valores más elevados en AfA, que decrecieron aguas abajo hacia S3 (Tabla 1, Figura 3). El patrón estacional fue

Tabla 1. Media y desvío estándar ( $n=3$ ) de los parámetros medidos en el Arroyo Carabassa (S) y sus afluentes (Af). $\mathrm{Nd}$ : niveles no detectables.

Table 1. Mean and standard deviation $(n=3)$ of the parameters measured in the Carabassa Stream (S) and tributaries (Af). Nd: undetectable levels

\begin{tabular}{|c|c|c|c|c|c|c|}
\hline & AfA & AfB & S1 & AfC & S2 & S3 \\
\hline Velocidad corriente $(\mathrm{m} / \mathrm{s})$ & $0.2 \pm 0.1$ & nd & $0.2 \pm 0.07$ & nd & $0.02 \pm 0.01$ & $0.06 \pm 0.06$ \\
\hline Caudal $\left(\mathrm{m}^{3} / \mathrm{s}\right)$ & $0.21 \pm 0.11$ & nd & $0.58 \pm 0.32$ & nd & $0.03 \pm 0.02$ & $0.37 \pm 0.41$ \\
\hline Temperatura $\left({ }^{\circ} \mathrm{C}\right)$ & $18.7 \pm 4.86$ & $17.63 \pm 5.18$ & $17.40 \pm 3.99$ & $15.77 \pm 4.1$ & $18.47 \pm 4.71$ & $16.70 \pm 5.8$ \\
\hline Sólidos totales $(\mathrm{mg} / \mathrm{L})$ & $1606.6 \pm 94.5$ & $603.3 \pm 220.5$ & $1430 \pm 156.2$ & $440 \pm 222.71$ & $943.3 \pm 297.4$ & $1076.7 \pm 357.3$ \\
\hline Turbidez (NTU) & $319.6 \pm 38.5$ & $147.67 \pm 30.5$ & $268 \pm 19.3$ & $187 \pm 20.22$ & $161.67 \pm 15.95$ & $152.3 \pm 6.1$ \\
\hline Conductividad $(\mu \mathrm{S} / \mathrm{cm})$ & $2413.3 \pm 171.5$ & $1165.7 \pm 499.8$ & $2253.3 \pm 195$ & $601.3 \pm 396.5$ & $1772.3 \pm 536.3$ & $1676.3 \pm 522.7$ \\
\hline $\mathrm{pH}$ & $8.24 \pm 0.41$ & $7.91 \pm 0.32$ & $8.23 \pm 0.27$ & $7.72 \pm 0.3$ & $8.17 \pm 0.21$ & $8.07 \pm 0.19$ \\
\hline Oxígeno disuelto $(\mathrm{mg} / \mathrm{L})$ & $2.84 \pm 3.08$ & $5.68 \pm 1.67$ & $2.74 \pm 1.86$ & $2.74 \pm 0.59$ & $4.75 \pm 0.52$ & $3.97 \pm 0.4$ \\
\hline $\mathrm{DBO}(\mathrm{mg} / \mathrm{L})$ & $113.3 \pm 64.3$ & $39.33 \pm 13.01$ & $92.83 \pm 49.76$ & $23 \pm 15$ & $73.67 \pm 50.64$ & $51.67 \pm 23.67$ \\
\hline $\mathrm{DQO}(\mathrm{mg} / \mathrm{L})$ & $393.7 \pm 18.4$ & $116.3 \pm 19.1$ & $309.7 \pm 21.6$ & $83.67 \pm 26.27$ & $213.7 \pm 125.1$ & $141.3 \pm 52.3$ \\
\hline $\mathrm{DBO} / \mathrm{DQO}$ & $0.29 \pm 0.16$ & $0.35 \pm 0.13$ & $0.29 \pm 0.14$ & $0.26 \pm 0.12$ & $0.34 \pm 0.06$ & $0.36 \pm 0.06$ \\
\hline $\mathrm{N}-\mathrm{NH}_{4}^{+}(\mathrm{mg} / \mathrm{L})$ & $18.62 \pm 22.39$ & $6.70 \pm 8.36$ & $18.06 \pm 22.38$ & $0.86 \pm 0.37$ & $10.53 \pm 3.61$ & $8.28 \pm 5.44$ \\
\hline $\mathrm{N}-\mathrm{NO}_{3}^{-}(\mathrm{mg} / \mathrm{L})$ & $11.32 \pm 3.02$ & $17.32 \pm 2.45$ & $12.20 \pm 1.92$ & $31.13 \pm 10.68$ & $12.94 \pm 2.24$ & $15.47 \pm 2.93$ \\
\hline $\mathrm{N}^{-\mathrm{NO}_{2}^{-}}(\mathrm{mg} / \mathrm{l})$ & $0.17 \pm 0.12$ & $1.29 \pm 1.99$ & $0.25 \pm 0.19$ & $2.24 \pm 3.08$ & $0.65 \pm 0.93$ & $1.02 \pm 1.58$ \\
\hline $\mathrm{NT}(\mathrm{mg} / \mathrm{L})$ & $109.9 \pm 20.5$ & $106.2 \pm 43.7$ & $88.17 \pm 45.25$ & $48.22 \pm 3.41$ & $88.9 \pm 14.2$ & $42.28 \pm 8.55$ \\
\hline $\mathrm{PT}(\mathrm{mg} / \mathrm{L})$ & $9.40 \pm 7.77$ & $7.17 \pm 3.79$ & $9.25 \pm 7$ & $2.28 \pm 1.29$ & $7.55 \pm 2.15$ & $5.74 \pm 2.91$ \\
\hline SAAM $(\mathrm{mg} / \mathrm{L})$ & $0.74 \pm 0.65$ & $0.05 \pm 0.09$ & $0.78 \pm 0.63$ & $0.09 \pm 0.09$ & $0.31 \pm 0.28$ & $0.28 \pm 0.21$ \\
\hline
\end{tabular}




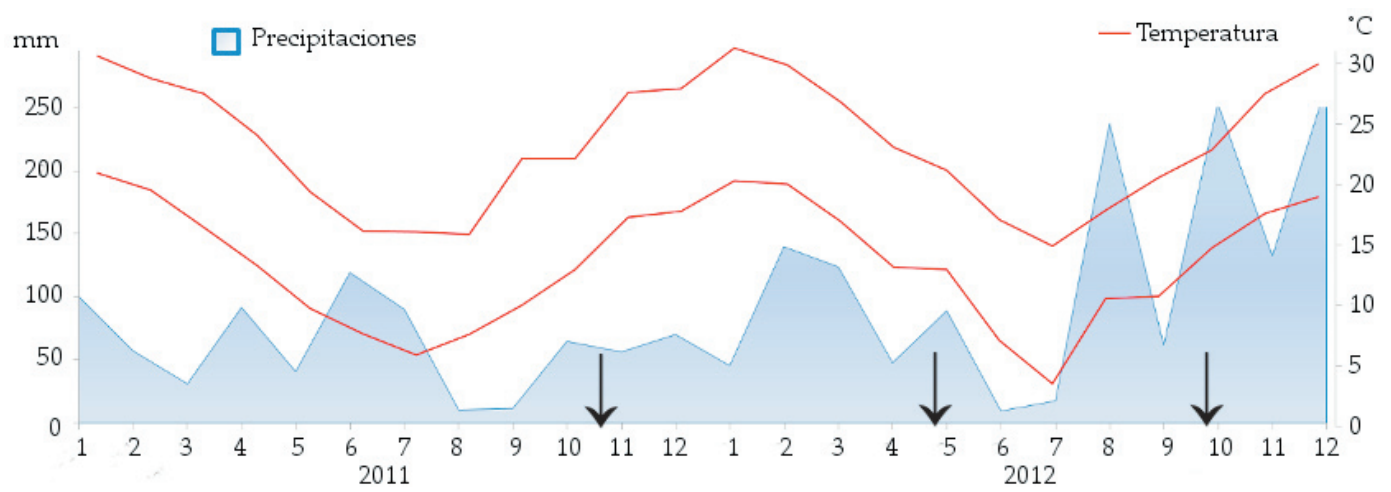

Figura 2. Precipitaciones registradas en mm por el Servicio Meteorológico Nacional (gráfico de área) y temperatura mensual máxima y mínima (gráfico de línea) para la ciudad de Pilar durante el período estudiado. Las flechas marcan las fechas de cada muestreo.

Figure 2. Monthly maximum and minimum air temperature (line graph) and rainfall (area graph) recorded for 2011 and 2012 in Pilar City. Arrows indicate sampling dates. Information provided by the Servicio Meteorológico Nacional (Argentina).

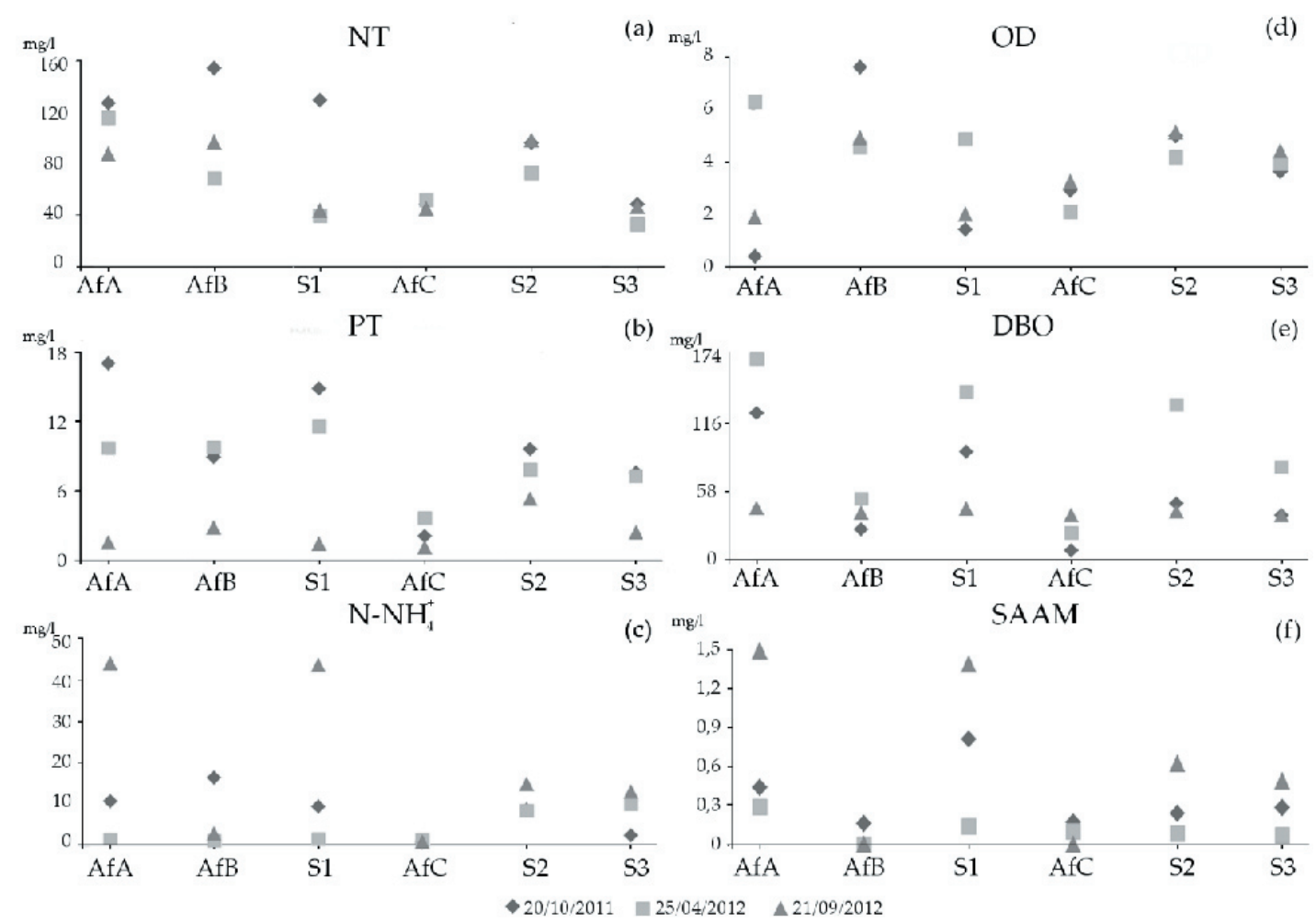

Figura 3. Variación espacio-temporal en el Arroyo Carabassa (S) y sus tributarios (Af) de: a) nitrógeno total (NT), b)

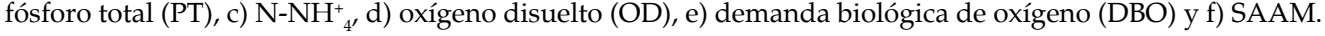

Figura 3. Spatio-temporal variation in the Carabassa Stream (S) and tributaries (Af) of: a) total nitrogen (NT), b) total phosphorus, c) ${\mathrm{N}-\mathrm{NH}_{4}^{+}}_{4}(\mathrm{FT}), \mathrm{d}$ ) dissolved oxygen (OD), e) biological oxygen demand (DBO) and f) SAAM.

coincidente para estos parámetros: en octubre de 2011 y abril de 2012 fueron más elevados y decrecieron en setiembre de 2012, con excepción de SAAM, que evidenció sus valores máximos en setiembre (Figura 3f).

Las concentraciones medias de $\mathrm{N}_{-} \mathrm{NO}_{3}^{-}$ fueron siempre elevadas $(>11 \mathrm{mg} / \mathrm{L})$ y se mantuvieron relativamente constantes desde AfA hasta S3. AfC presentó los valores más altos.
Sin embargo, las concentraciones de las especies más reducidas del nitrógeno mostraron diferencias estacionales. El N-NO- aumentó de forma marcada en el otoño de 2012 por pulsos en AfB y AfC (datos no mostrados), mientras que el $\mathrm{N}-\mathrm{NH}^{+}{ }_{4}$ creció debido al pico en AfA en octubre de 2012, en coincidencia con el patrón espacio-temporal de las SAAM (Figura 3c). Las concentraciones de NT fueron elevadas en AfA y AfB hasta la naciente del 
arroyo, se verificó una disminución aguas abajo y un leve repunte en S2 (Figura 3a). Las concentraciones de PT fueron muy elevadas, especialmente en AfA y AfB y S1 en octubre y abril, y disminuyeron aguas abajo hasta S3 (Figura 3b). Las concentraciones de OD fueron generalmente bajas (Tabla 1, Figura 3d) y presentaron diferencias estacionales. En los períodos cálidos (primavera 2011 y 2012), los tenores bajos de AfA se mantuvieron en S1, mientras que en otoño los valores fueron mayores en ambos sitios. Cabe destacar que los valores en S2 y S3 se mantuvieron próximos a $4 \mathrm{mg} / \mathrm{L}$. La DBO, consistentemente mayor en otoño 2012 y menor en setiembre 2012, presentó un patrón espacial idéntico a lo largo del tiempo, con máximos en AfA, los cuales impactaron en particular en la naciente del Arroyo Carabassa (Figura 3e). El patrón espacial de la DQO (datos no mostrados) fue similar a la DBO $(r=0.80, P<0.05)$, aunque con un leve repunte de los valores en S2 en otoño 2012 que minimizó las diferencias temporales de esta variable.

Según los valores del ICA, el arroyo presentó estados desde polución intermedia a muy elevada (Tabla 2). En AfC se obtuvieron los valores más altos $(\mathrm{ICA}=5)$, en tanto que el resto de los sitios presentó un promedio de ICA=3. En octubre de 2011 se midió el mayor deterioro, evidenciado por valores entre 1 (mínimo calculado para AfA) y 2. AfC constituyó una excepción, ya que permaneció con indicadores de calidad de agua más altos.

El ordenamiento de las muestras realizado con un ACP con las variables físicas, químicas e hidrológicas explicó, entre los dos primeros ejes, $57.8 \%$ de la varianza total. El primer factor acumuló 35.7\% de la varianza, con una correlación positiva con SST ( $\mathrm{r}=0.84)$, DQO $(\mathrm{r}=0.81)$ y SAAM $(\mathrm{r}=0.72)$ y negativa con $\mathrm{N}$ $\mathrm{NO}_{3}^{-}(\mathrm{r}=-0.68)$. El segundo eje explicó $22.1 \%$ de la variación y se correlacionó de forma positiva con el PT (r=0.74) y negativa con el N-NH${ }_{4}^{+}$ $(r=-0.62)$. Los sitios se ordenaron según el eje 1 en función de la calidad del agua. Así, S1 y AfA se asociaron a valores altos de DQO, SST y SAAM. Cabe destacar que la DBO no fue incluida en el ACP por presentar una correlación alta con la DQO. A su vez, AfC se asoció a las concentraciones bajas de estos parámetros y a las más elevadas de ${\mathrm{N}-\mathrm{NO}_{3}^{-}}_{3}$ (Figura 4).

\section{Análisis de microorganismos}

En general, las bacterias coliformes totales y fecales mostraron una abundancia elevada (Tabla 2), con valores que oscilaron entre 160 y $22000 \mathrm{NMP} / 100 \mathrm{~mL}$, lo que supera el nivel guía de 1000 NMP/100 mL (SRHN 2005). En abril de 2012, en coincidencia con los menores niveles hídricos, se estimaron las abundancias más altas y se registraron los valores máximos en S1.

La densidad fitoplanctónica total fluctuó de forma marcada entre los afluentes y entre los períodos de muestreo, en especial en el $\mathrm{AfB}$, donde alcanzó un valor máximo de 1400400 individuos/mL y un mínimo de 10300 individuos/mL (Tabla 2). En el curso principal del Arroyo Carabassa se detectaron variaciones menos marcadas y sin un patrón espacial consistente en los distintos períodos

Tabla 2. Media y desvío estándar correspondiente a las tres fechas de muestreo ( $\mathrm{n}=3$ ) de la abundancia de bacterias coliformes totales (CT) y fecales (CF) y fitoplancton, grupos algales dominantes y codominantes, Índice de Calidad de Agua (ICA) y porcentaje de euglenofitas con alteraciones (septiembre 2012) en el Arroyo Carabassa (S) y sus tributarios (Af).

Table 2. Mean and standard deviation corresponding to the sampling dates $(n=3)$ of bacteria (total and fecal coliforms, $\mathrm{CT}$ and CF) and phytoplankton abundance, dominant and co-dominant groups, and ICA index values and percentage of altered euglenoids (September 2012) in the Carabassa Stream (S) and tributaries (Af).

\begin{tabular}{|c|c|c|c|c|c|c|}
\hline & AfA & AfB & AfC & S1 & S2 & S3 \\
\hline $\begin{array}{l}\text { Bacterias CT } \\
(\mathrm{NMP} / 100 \mathrm{~mL})\end{array}$ & $11066 \pm 5460$ & $2613 \pm 3629$ & $7760 \pm 11475$ & $9667 \pm 10815$ & $5267 \pm 3700$ & $6020 \pm 9516$ \\
\hline $\begin{array}{l}\text { Bacterias CF } \\
(\mathrm{NMP} / 100 \mathrm{~mL})\end{array}$ & $2233 \pm 1078$ & $500 \pm 781$ & $1447 \pm 2212$ & $2193 \pm 2622$ & $943 \pm 886$ & $1287 \pm 1917$ \\
\hline $\begin{array}{l}\text { Fitoplancton } \\
\text { total (ind/mL) }\end{array}$ & $678406 \pm 272508$ & $493728 \pm 785389$ & $37443 \pm 5916$ & $568386 \pm 431765$ & $581178 \pm 78653$ & $280676 \pm 243282$ \\
\hline $\begin{array}{l}\text { Grupo } \\
\text { dominante }\end{array}$ & Chlorophyta & Chlorophyta & Chlorophyta & Chlorophyta & Chlorophyta & Chlorophyta \\
\hline $\begin{array}{l}\text { Grupo } \\
\text { codominante }\end{array}$ & $\begin{array}{l}\text { Cyanobacteria } \\
\text { Bacillariophyta }\end{array}$ & $\begin{array}{c}\text { Bacillariophyta } \\
\text { Euglenophyta }\end{array}$ & $\begin{array}{c}\text { Bacillariophyta } \\
\text { Cryptophyta }\end{array}$ & $\begin{array}{l}\text { Cyanobacteria } \\
\text { Euglenophyta }\end{array}$ & $\begin{array}{l}\text { Cyanobacteria } \\
\text { Euglenophyta }\end{array}$ & $\begin{array}{l}\text { Cyanobacteria } \\
\text { Euglenophyta }\end{array}$ \\
\hline $\begin{array}{l}\text { Euglenofitas } \\
\text { totales (ind/mL) }\end{array}$ & $8504 \pm 14151$ & $2501 \pm 3200$ & $11428 \pm 16220$ & $2592 \pm 2976$ & $5900 \pm 125$ & $1562 \pm 785$ \\
\hline $\begin{array}{l}\text { Euglenofitas } \\
\text { anómalas }\end{array}$ & $100 \%$ & $13 \%$ & $8 \%$ & $95 \%$ & $87 \%$ & $81 \%$ \\
\hline ICA & $3 \pm 1$ & $3 \pm 0.6$ & $5 \pm 0.6$ & $3 \pm 0.7$ & $3 \pm 0.2$ & $3 \pm 0.1$ \\
\hline
\end{tabular}




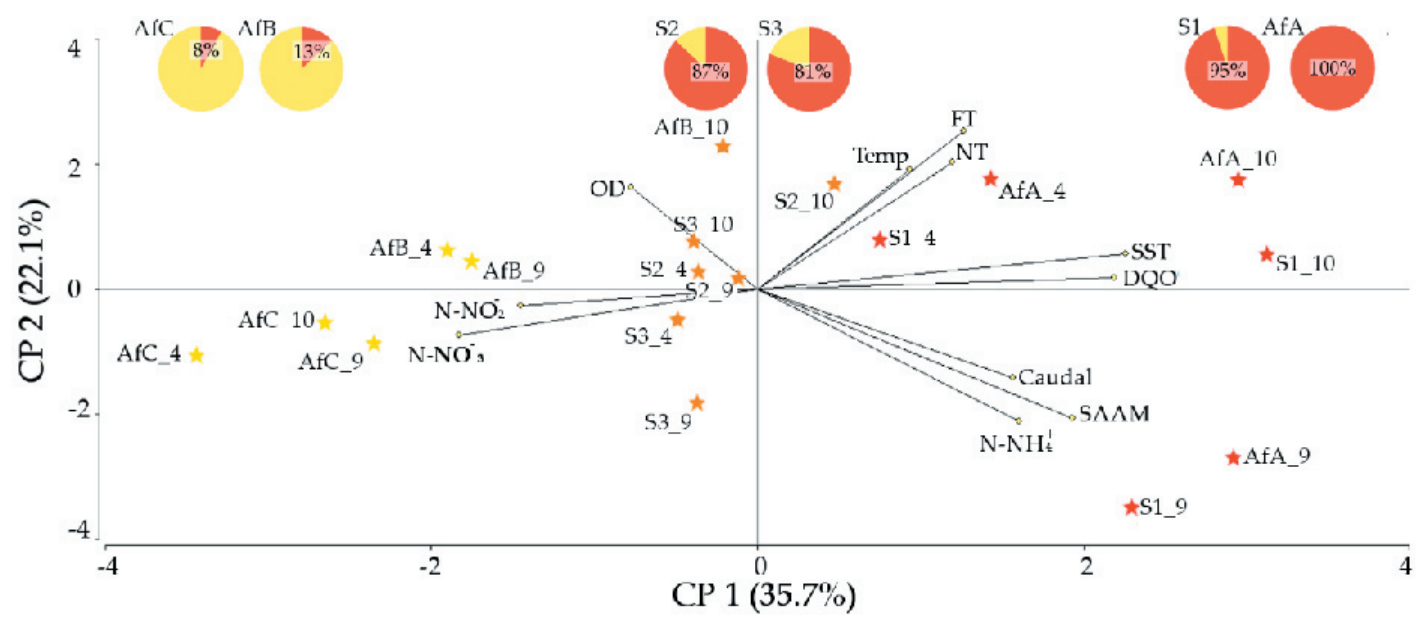

Figura 4. Representación del análisis de componentes principales basado en los parámetros físicos y químicos de las muestras del cauce principal del Arroyo Carabassa (S1,S2, S3) y de los afluentes (AfA, AfB, AfC), indicando los meses analizados $(4,9$ y 10$)$. Las estrellas rojas señalan el mayor grado de deterioro con respecto al ordenamiento resultante; las naranjas, el deterioro intermedio, y las amarillas, el más leve. En la parte superior de la imagen se observan los porcentajes de euglenofitas con deformaciones morfológicas (rojo) detectados en el muestreo de septiembre del 2012 en el Arroyo Carabassa (S) y tributarios (Af).

Figure 4. Plot of the principal component analysis based on the chemical and physical parameters from samples of the Carabassa Stream (S1, S2, S3) and tributaries (AfA, AfB, AfC), indicating the months $(4,9$ and 10) in each label. The color of the stars represent different water quality: red, highest pollution; orange, medium pollution, and yellow, lowest pollution. At the top of the image appears the percentage of Euglenophyta with anomalous morphology (red) observed at the Carabassa Stream (S) and its tributaries (Af) on September 2012.

Tabla 3. Lista de especies de euglenofitas encontradas en los sitios de muestreo del Arroyo Carabassa (S) y de los tributarios (Af). Las letras indican los meses: (O) octubre de 2011, (A) abril de 2012, (S) septiembre de 2012.

Table 3. List of Euglenophyta species found at each sampling site of the Carabassa Stream (S) and its tributaries (Af). Letters indicate the months: (O) October 2011, (A) April 2012, (S) September 2012.

\begin{tabular}{|c|c|c|c|c|c|c|}
\hline Especies & AfA & AfB & S1 & AfC & S2 & S3 \\
\hline Euglena agilis & A & & & & & \\
\hline E. anabaena & & $S$ & & & & \\
\hline E. clara & & & S & & & \\
\hline E. clavata & A & & & & A & \\
\hline E. ehrenbergii & $\mathrm{O}$ & & & A & A & \\
\hline E. intermedia & & & & & & $S$ \\
\hline E. intermedia var. klebsii & & & & S & & \\
\hline E. pisciformis & & & & $S$ & & \\
\hline Lepocinclis acus & OA & S & AS & OAS & AS & Os \\
\hline L. fusiformis & $\mathrm{S}$ & & & & & \\
\hline L. ovum & $\mathrm{A}$ & & & & & \\
\hline L. oxyuris & A & A & OAS & OAS & Os & \\
\hline L. playfairiana & A & & A & A & A & A \\
\hline L. texta var. richiana & & & & & & S \\
\hline L. tripteris & & & & OA & & \\
\hline Phacus anomalus & & & & S & & \\
\hline P. curvicauda & & & & S & & \\
\hline P. longicauda & AOS & A & AS & AOS & OAS & OAS \\
\hline P. onyx & & S & & & & \\
\hline P. texta & $\mathrm{O}$ & & & & & \\
\hline P. tortus & $\mathrm{OA}$ & & AS & OAS & OS & $\mathrm{O}$ \\
\hline Strombomonas bacillifera var. minima & & & & $\mathrm{O}$ & & \\
\hline S. brevicaudata & & & & $\mathrm{O}$ & & \\
\hline S. scabra & A & & & OAS & & \\
\hline S. scabra var. coberensis & A & & & & & \\
\hline S. scabra var. longicollis & & & & & S & \\
\hline Trachelomonas granulata & & & & & & S \\
\hline T. lefevrei & & S & & $\mathrm{O}$ & & \\
\hline
\end{tabular}


analizados. Sólo en aguas bajas (abril de 2012) se evidenció una tendencia creciente de las abundancias hacia S3. Con respecto a la composición del fitoplancton, se registró una dominancia de clorofitas en la mayoría de los sitios, con alternancia o co-dominancia de cianobacterias y euglenofitas (Tabla 2). En los afluentes la diversidad de los grupos fue generalmente mayor respecto a la detectada en el cauce principal.

La abundancia de euglenofitas se correlacionó con la concentración de amonio $(\mathrm{r}=0.56, P<0.05)$ y la presencia de SAAM $(\mathrm{r}=0.5$, $P<0.05)$. Además, se observó que la proporción de euglenofitas que componen el fitoplancton total se correlacionó de forma inversa con la nueva variable descriptiva de la calidad del agua del sistema (obtenida como el mayor autovalor del ACP) $(r=-0.54, P<0.05)$. Las especies de euglenofitas observadas en cada muestreo se detallan en la Tabla 3. Durante el muestreo de setiembre de 2012, este grupo mostró su mayor riqueza específica, y fueron los afluentes los que presentaron una mayor diversidad. En esta ocasión también
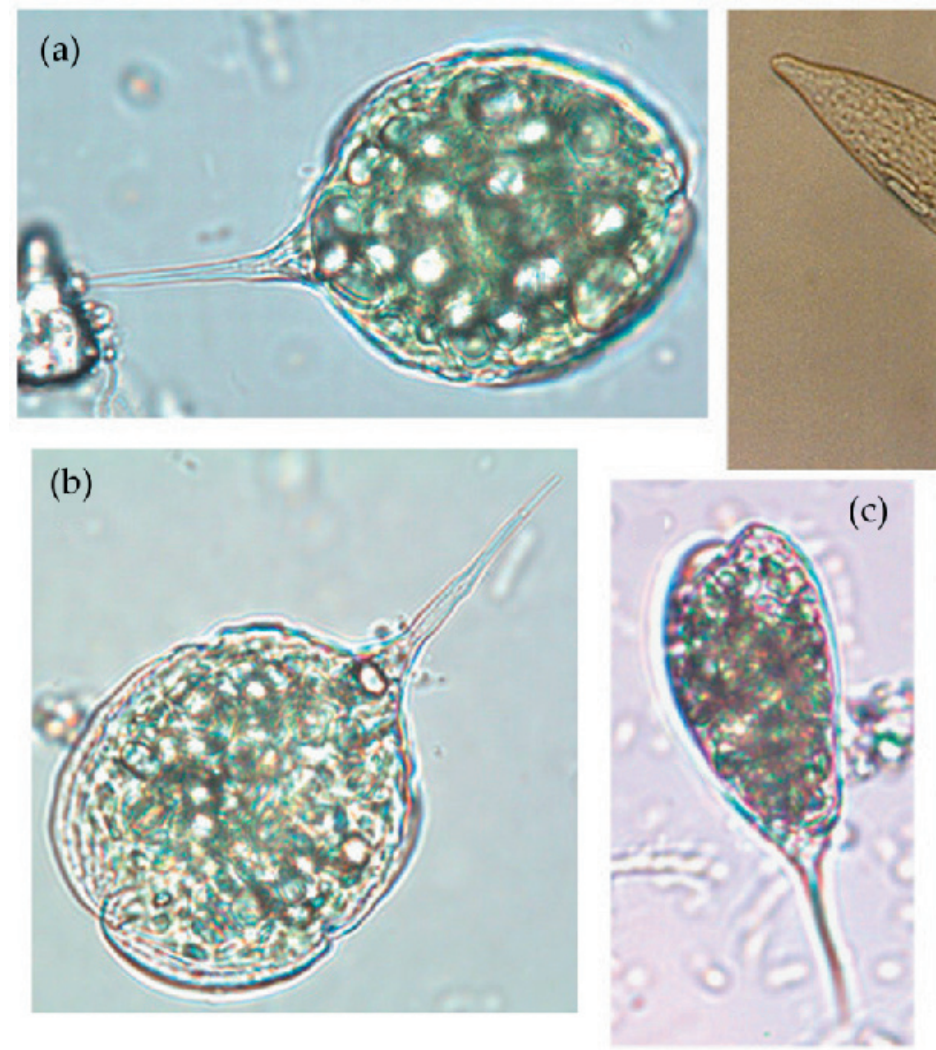

se encontró un gran porcentaje de estas algas con claras anomalías morfológicas, en coincidencia con un patrón creciente de DQO, DBO, SST y SAAM (Figura 4, Tabla 2). En AfA, el 100\% de los organismos presentó una morfología anómala; sin embargo, esta proporción disminuyó aguas abajo en el Arroyo Carabassa. Contrariamente, en AfB y AfC, estos porcentajes se invirtieron y mostraron una mayor abundancia de organismos sin alteraciones. Las SAAM se correlacionaron con el número de euglenofitas anómalas $(\mathrm{r}=0.76, P<0.05)$. Los especímenes de Lepocinclis acus presentaron mayor ancho celular, menor longitud y gran acumulación de sustancias de reserva (Figura 5d). También se detectaron numerosos ejemplares de Phacus longicauda (Figura 5a-c), con un incremento marcado en sus dimensiones y en la cantidad y forma de sus granos de paramilon. Estos cuerpos, que normalmente se presentan como discos aplanados, aparecían muy voluminosos y, en algunos casos, con forma de carretel, lo que determinaba un engrosamiento celular dorsiventral. Algunos especímenes presentaron muescas en el contorno celular.

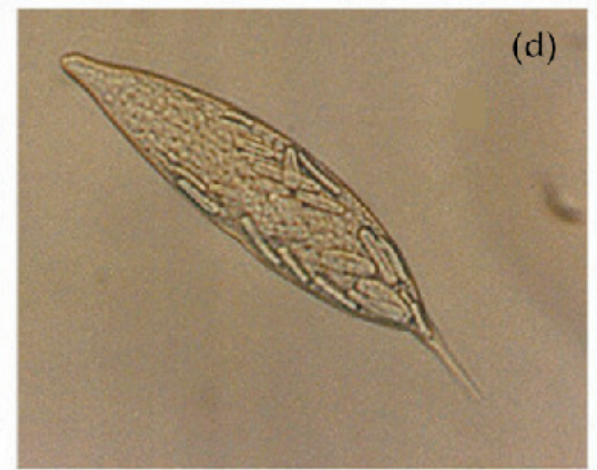

c)

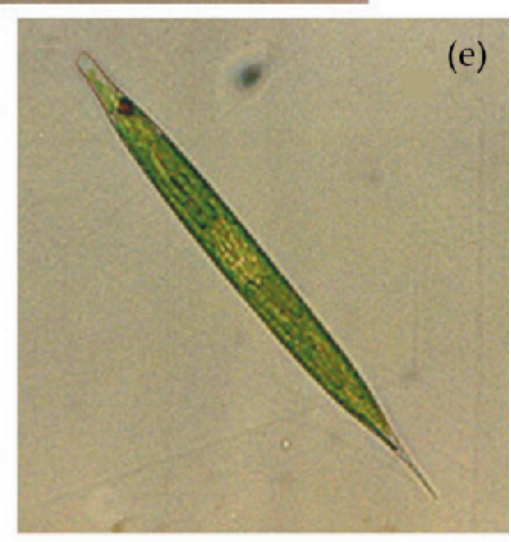

Figura 5. Euglenofitas que presentaron alteraciones respecto de su morfología habitual. a-b) Phacus longicauda, vista frontal, c) Phacus longicauda, vista lateral, d) Lepocinclis acus, morfología alterada y e) Lepocinclis acus, morfología habitual.

Figure 5. Euglenophyta presenting alterations respect regular morphology. a-b) Phacus longicauda, frontal view, c) Phacus longicauda, lateral view, d) Lepocinclis acus, anomalous morphology and e) Lepocinclis acus, regular morphology. 
En numerosos ejemplares de Lepocinclis acus de AfB y S1 se detectó una menor relación longitud/ancho y gran número de granos de paramilon pero sin cambios del espesor celular.

\section{Discusión}

Los cursos de agua de escaso caudal que fluyen por las llanuras próximas a los centros urbanos e industriales están expuestos a una fuerte presión antrópica, con graves consecuencias en la calidad del agua y en sus comunidades acuáticas. La polución orgánica en el Arroyo Carabassa es muy elevada, lo cual es un ejemplo claro de la vulnerabilidad de los arroyos pampeanos periurbanos bonaerenses. El sistema estudiado se caracteriza por la presencia de concentraciones elevadas de nutrientes, materia orgánica y sólidos en suspensión, que se asocian a bajos tenores de OD, y otros contaminantes tales como las SAAM. Las concentraciones de materia orgánica (estimada según las DBO y DQO), nutrientes, OD y la abundancia de bacterias coliformes superaron los valores establecidos en la legislación argentina para proteger la vida acuática (SRHN 2005). El efecto de la polución orgánica y la eutrofización son determinantes de las diferencias existentes entre arroyos pampeanos (Feijoó and Lombardo 2007). Estos arroyos están sujetos a variaciones fuertes en sus concentraciones de fósforo y de material particulado (Rodríguez Castro 2015; Ranieri 2015) a causa de las fluctuaciones estacionales y las precipitaciones (Feijoó et al. 1999). Si bien los nutrientes elevados se asocian a aumentos de ingresos desde fuentes puntuales y no puntuales, tales como las que afectan a este curso, también actuarían las tasas de remoción menores descriptas para arroyos urbanizados con funciones ecosistémicas alteradas (Meyer et al. 2005).

\section{Calidad del agua}

El déficit de saturación del oxígeno en ríos someros y con gran carga de materia orgánica es frecuente (Blesama et al. 2012). En este caso, el efecto generado por las descargas urbanas e industriales empeoraron esta condición. Los valores del cociente $\mathrm{DBO} / \mathrm{DQO}$ entre 0.1-0.4 indican que una gran proporción de los contaminantes que ingresan en el curso de agua no son biodegradables (Miller et al. 1996). A su vez, debido a la falta de oxígeno o a que el tiempo o la distancia transcurridos entre los sitios fueron insuficientes, mucho del material biodegradable aportado no habría sido descompuesto en su totalidad y se acumuló en el ambiente. Al comparar los valores de $\mathrm{DQO}, \mathrm{N}^{-\mathrm{NO}^{-}}{ }^{\prime}, \mathrm{N}^{-} \mathrm{NO}_{2}^{-}$y N-NH${ }_{4}^{+}$ del Arroyo Carabassa con los publicados por Di Marzio et al. (2008), provenientes de efluentes fabriles de la región, se detectó una gran similitud, lo cual señalaría la gran influencia de las descargas industriales en este curso, especialmente en AfA. El análisis de ordenamiento espacial según las variables físicas y químicas (Figura 4) indicó la existencia de un gradiente longitudinal de calidad de agua creciente desde uno de los afluentes (AfA) que da origen al Arroyo Carabassa hasta su confluencia con el río Luján (S3). Así, en AfA y en $\mathrm{S} 1$ se registraron las concentraciones más elevadas de la mayoría de los parámetros físicos y químicos indicadores de contaminación (SST, DBO, DQO, SAAM y N-NH${ }_{4}^{+}$), en tanto que aguas abajo su valor disminuyó. Los afluentes AfB y AfC se ordenaron en el extremo del gradiente que corresponde a aguas menos deterioradas según estos parámetros. El impacto negativo en la calidad del agua del afluente donde la industria láctea vierte sus efluentes evidencia que su tratamiento sería insuficiente. Además, la detección de altas concentraciones de SAAM, normalmente utilizadas como detergente para limpieza de las instalaciones del rubro alimentario, refuerza esta idea. Los otros dos afluentes mostraron un deterioro menor, a pesar de estar afectados por un frigorífico y por una urbanización, respectivamente.

El grado de deterioro del Arroyo Carabassa y sus afluentes se evidenció en los valores del ICA, que se corresponden con una polución intermedia (AfC) a elevada (S1S3, AfA y AfC). En consecuencia, los valores de varios parámetros incumplen los niveles guía nacionales o internacionales. Las concentraciones promedio de SAAM superaron en cuatro sitios los límites establecidos para la preservación de la vida acuática (0.1 mg/L) (DOF 1989). Las concentraciones de N-NO-2 y $\mathrm{N}-\mathrm{NH}^{+}{ }_{4}$ casi siempre superaron los valores establecidos (0.06 mg/L y $1.37 \mathrm{mg} / \mathrm{L}$, respectivamente) para la protección de la vida acuática en cuerpos de agua continentales (Ley 24.051 de Residuos Peligrosos, Decreto 831/93). En la mayoría de los sitios, las concentraciones de oxígeno disuelto fueron inferiores al nivel guía considerado crítico para la protección de la vida acuática (5 mg/L), mientras que los valores de $\mathrm{DBO}$ superaron el nivel guía (3 $\mathrm{mg} / \mathrm{L}$ ) en el $89 \%$ de las mediciones realizadas (SRHN 2005). 


\section{Comunidad microbiana}

La falta de vegetación riparia y la alta irradiancia en las cabeceras y en arroyos de dimensiones medianas de las cuencas hídricas pampeanas, caracterizadas por sus bajos caudales, la ausencia de períodos secos y temperaturas extremas, permiten una producción primaria autóctona sustentada por las comunidades algales o de macrófitas con relaciones de producción/respiración $>1$ (Vilches and Giorgi 2010). Las concentraciones elevadas de PT y NT registradas permiten asumir que en el Arroyo Carabassa la producción autóctona por algas o macrófitas es considerable, tal como lo explica Dodds (2007) en función de la cantidad sustancial de energía basal que reciben los cursos desde fuentes terrestres (alóctonas). La influencia de los factores de control negativo (e.g., la remoción por lavado o la depredación de organismos fotosintéticos) explicaría la disminución de la abundancia fitoplanctónica a lo largo del curso (octubre 2011 y setiembre 2012). Sólo en períodos de bajo caudal y luego de la estación cálida (abril de 2012) se verifica un aumento de la densidad fitoplanctónica aguas abajo en el Arroyo Carabassa. El sistema sustenta densidades fitoplanctónicas elevadas, con dominancia de clorofitas, que alternaron con aumentos de cianobacterias inferiores al 20\% de la abundancia, y de euglenofitas. Gómez and O'Farrell (2014) describieron una alternancia similar de clases algales en el tramo medio del río Luján, donde comenzaba a acentuarse la polución industrial y urbana. La contribución de las euglenofitas al fitoplancton, según la correlación con la variable descriptiva de la calidad de agua $(\mathrm{ACP})$, aumentó levemente en los sitios con menor deterioro (AfC y AfB), en coincidencia con valores de los parámetros medidos que no superaron o resultaron levemente mayores a los reglamentados por las normas nacionales o extranjeras consultadas. En estos sitios también se encontró un mayor número de especies de euglenofitas y la codominancia de grupos algales.

La relación encontrada entre la calidad de agua y las euglenofitas indicaría que la polución orgánica generaría las alteraciones morfológicas observadas en los individuos de distintas especies. Los cambios observados en Lepocinclis acus y Phacus longicauda coinciden con los observados en algunas especies por Conforti (1998), Bauer et al. (2012), Nannavecchia et al. (2014) y Nannavecchia 2016. En estos trabajos, las modificaciones se explican como respuesta al exceso de materia orgánica en el ambiente, lo cual coincide con las características generales del sistema estudiado. Conforti (1998) llama la atención sobre el hecho de que las células se deforman a pesar de ser especies que presentan películas rígidas. Según estos estudios, los cambios pueden llegar hasta un cierto límite de alteración, luego del cual se produce la lisis celular y la liberación de los cuerpos de paramilon al medio. Este último hecho explicaría los numerosos granos de paramilon observados en algunas muestras de nuestro estudio.

El manejo de los temas relacionados a la calidad de agua de los arroyos se ha basadoen el conocimiento del funcionamiento de sistemas localizados en regiones templadas frías con un fuerte componente alóctono, aportado por los bosques deciduos marginales (Dodds 2007). Esto implicó que los bioindicadores de uso más común para evaluar los problemas asociados con el vertido de efluentes no tratados con alto contenido de materia orgánica fueran los ensambles de macroinvertebrados (Hellawell 1986), muy bien caracterizados para sistemas heterotróficos en donde el cauce está sombreado por la vegetación ribereña. Este trabajo pone de manifiesto la importancia de las comunidades autotróficas en arroyos pampeanos donde la luz no suele ser limitante para los productores primarios, e introduce la posibilidad de implementar el uso de los ensambles de euglenofitas como centinelas de los cambios provocados por el exceso de materia orgánica característico de cursos urbanizados.

\section{Conclusión}

El Arroyo Carabassa y sus afluentes revelaron un alto deterioro, evidenciado por una eutrofización elevada, con una severa carga orgánica e indicadores de contaminación fecal, consecuencia de la actividad antrópica en la cuenca. El uso de índices sencillos que involucran pocas variables físicas y químicas (ICA) en combinación con monitoreos de ensambles fitoplanctónicos resultaron eficientes para evaluar los cambios espaciales y temporales en los niveles de contaminación orgánica de este arroyo de la llanura pampeana. La mayor frecuencia de anomalías morfológicas de euglenofitas en sitios poluidos indica la potencialidad de este atributo como centinela de contaminación orgánica en cursos autótrofos. 
Agradecimientos. Al Laboratorio de Agua y Ambiente de la Asociación Bonaerense de Investigaciones Ambientales por su colaboración, al Servicio Meteorológico
Nacional y al financiamiento otorgado a la Dra. Conforti PIP CONICET, 2010-2012, №283 y 2014-2016, $\mathrm{N}^{\circ}$ 11220130100674; UBACYT 2014-2017. 20020130100201BA.

\section{REFERENCIAS}

APHA. American Public Health Association. 2005. Standard Methods for the Examination of Waters and Wastewaters, 19th ed., Washington, DC.

Bauer, D. E., V. Conforti, L. Ruiz, and N. Gómez. 2012. An in-situ test to explore the responses of Scenedesmus acutus and Lepocinclis acus as indicators of the changes in water quality in lowland streams. Ecotoxicology and Environmental Safety 77:71-78.

Berón, L. 1984. Evaluación de la calidad de las aguas de los ríos de la Plata y Matanza - Riachuelo mediante la utilización de índices de calidad de agua. Ministerio de Economía. Buenos Aires, Argentina.

Blesama, A., M. Dos Santos Afonso, and C. Apellam. 2012. Agua y ambiente, un enfoque desde la química. Eudeba. Buenos Aires, Argentina.

Ciugulea, I., and E. Triemer. 2010. A color atlas of photosynthetic euglenoids, Michigan State University Press, East Lansing.

Conforti, V. 1998. Morphological changes of Euglenophyta in response to organic enrichment. Hydrobiologia 369/370: 277-285.

Conforti, V. 1999. A taxonomic and ultrastructural study of Trachelomonas Ehr. (Euglenophyta) from subtropical Argentina, Cryptogamie Algologie 20(3):167-207.

Conforti, V., and G. J. Joo. 1994. Taxonomic and ultrastructural study of Trachelomonas Ehr. and Strombomonas Defl. (Euglenophyta) from Oxbow Lakes in Alabama and Indiana (U.S.A.). Cryptogamie Algologie 15:267-286.

Di Marzio, W. D., M. E. Sáenz, and J. L. Alberdi. 2008. Evaluación de la ecotoxicidad de efluentes industriales y municipales, caso de estudio Río Luján - Región Ciudad de Pilar. Ingeniería sanitaria y ambiental 100:102-105.

Dodds, W. K. 2007. Trophic state, eutrophication and nutrient criteria in streams.Trends in ecology and evolution 22: 669-676.

DOF. Diario Oficial de la Federación. Acuerdo por el que se establecen los Criterios Ecológicos de Calidad del Agua. CE-CCA-001/89. México.

Feijoó, C. S., A. Giorgi, M. García, and F. Momo.1999. Temporal and spatial variability in streams of a pampean basin. Hydrobiologia 394:41-52.

Feijoó, C. S., and R. J. Lombardo. 2007. Baseline water quality and macrophyte assemblages in Pampean streams: A regional approach. Water Research 41(7):1399-1410.

Fidalgo, F. 1983. Algunas características de los sedimentos superficiales en la cuenca del río Salado y en la Pampa ondulada. Pp. 1045-1066 en Coloquio Internacional sobre Hidrología de Grandes Llanuras.

Giorgi, A. 2001. Cost of remediation of the Lujan River (Argentina). Transactions on Ecology and the Environment 46.

Gómez, N., and I. O'Farrell. 2014. Phytoplankton from urban and suburban polluted rivers. Advances in Limnology 65:127-142.

Hellawell, J. M. 1986. Biological indicators of freshwater pollution and environmental management. Elsevier, New York.

Herrero, A. C., and L. Fernández. 2008. Presión industrial en las Cuencas Metropolitanas de Buenos Aires. Pp. 113124 en De los ríos no me río, Diagnóstico y reflexiones sobre las Cuencas Metropolitanas de Buenos Aires. Temas, Buenos Aires, Argentina.

Hüber-Pestalozzi, G. 1955. Das phytoplankton des Susswasser, in A. Thienemann, Die Binnengewasser. 16(4):606

Kim, H. G., W. J. Choi, Y. G. Jung, C. S. Jung, J. S. Park, K. H. An, and C. I. Baek. 1999. Initiation of Cochlodinium polykrikoides blooms and its environmental characteristics around the Narodo island in the western part of South Sea of Korea. Bulletin of the National Fisheries Research and Development Institute, Korea 57:119-129.

Meyer J. L., M. J. Paul, and K. Taulbeew. 2005. Stream ecosystem function in urbanizing landscapes. Journal of North American Benthological Society 4(3):602-612.

Miller, M., R. L. Valentine, M. Roehl, and P. J. J Álvarez. 1996. Chemical and microbiological assessment of pendimenthalin-contaminated soil after treatment with Fenton's reagent. Water Research 30(11):2579-2586.

Momo, F., A. Zalts, E. Hughes, A. Ventura, T. Macro, H. Ceretti, and S. Ramírez. 2003. Estado ecológico de la cuenca del río Luján y utilidad de los indicadores biológicos para su control. En Las aguas bajan turbias. CoEd, Al Margen y Universidad Nacional de General Sarmiento, Buenos Aires, Argentina.

Nannavecchia, P., A. Tolivia, and V. Conforti. 2014. Ultrastructural alterations in Phacus brachykentron (Euglenophyta) due to excess of organic matter in the culture medium. Ecotoxicology and Environmental Safety 101:36-41.

Nannavecchia, P. 2016. Contaminación antrópica de los cuerpos de agua. Estudio de su efecto sobre organismos del fitoplancton a escala ecológica y de bioensayos de laboratorio. Tesis Doctoral. Facultad de Ciencias Exactas y Naturales. Universidad de Buenos Aires, Argentina. Pp. 218.

O'Farrell, I., R. Lombardo, P. De Tezanos Pinto, and C. López. 2002. The assessment of water quality in the Lower Luján River (Buenos Aires, Argentina): phytoplankton and algal bioassays. Environmental Pollution 120(2):207-218. 
Peluso, M. L., A. Salibián, and A. E. Ronco. 2016. Esquema para la categorización de la peligrosidad de sedimentos de fondo contaminados de sistemas fluviales. Limnetica 35(1):159-174.

Plataroti, M. C. 2010. Caracterización de la calidad del agua de una sección del Río Luján: Efectos sobre el fitoplancton. Tesis de licenciatura. Facultad de Ciencias Exactas y Naturales, Universidad de Buenos Aires, Argentina. Pp. 4748.

Popova, T. G. 1966. Evglenovyje vodoroski Vyp. 1. Flora Sporovych Rastenij. Izd. Nauka, Moscú.

Ranieri, M. 2015. Escalas de variación de la diversidad de macrófitas en arroyos de la provincia de Buenos Aires. Tesis Doctoral. Facultad de Ciencias Exactas y Naturales, Universidad de Buenos Aires, Argentina. Pp. 210.

Rodríguez, A., C. Ruggierio, and L. Fernández. 2008. Actividades productivas en la cuenca del río Luján. Su impacto sobre el agua. CEDet, Blanco Sobre Negro (5).

Rodríguez Castro, M. C. 2015. Capacidad de depuración de sustancias bioaprovechables en arroyos de llanura y su relación con el arsénico. Tesis Doctoral. Facultad de Ciencias Exactas y Naturales, Universidad de Buenos Aires, Argentina. Pp. 141.

Starmach, K. 1983. Euglenophyta; Eugleniny. Warszawa: Panstwowe Wydawnictwo Naukowe 3:1-594.

SRHN, Subsecretaría de Recursos Hídricos de la Nación. 2005. Niveles Guía Nacionales de Calidad de Agua Ambiente. República Argentina.

Tell, G., and V. Conforti. 1986. Euglenophyta pigmentadas de la Argentina. Pp. 301 en J. Cramer (ed.). Bibliotheca Phycologica.

UNESCO. 2006. Water a shared responsibility, United Nations World Water Development Report 2.7, Berghan Books, New York and UNESCO, Paris.

USEPA. Enviromental Protection Agency, Office of water. 1999. Nutrient criteria technical guidance manual: Rivers and streams. EPA- 822-D-99-003. Washington, DC.

Utermöhl, H. 1958. Zur Vervollkommung der quantitativen Phytoplankton-methodik. Mitteilung der Internationale Vereinigung für Theoretische unde Amgewandte Limnologie 9:1-38.

Vilches, C., and A. Giorgi. 2010. Metabolism in a macrophyte-rich stream exposed to flooding. Hydrobiologia 654: 57-65.

Venrick E. 1978. How many cells to count? Pp. 167-180 en Phytoplankton Manual, Monographs on oceanographic methodology. UNESCO Press, Paris.

Wolowski, K., and F. Hindák. 2005. Atlas of Euglenophytes. VEDA, Bratislava, Eslovaquia. Pp 136. 